\title{
Adaptabilidade e estabilidade de cultivares de soja em cinco épocas de plantio no cerrado de Rondônia(1)
}

\author{
Eloi Elias do Prado(2), Dario Minoru Hiromoto(3), Vicente de Paulo Campos Godinho( ${ }^{(2)}$, \\ Marley Marico Utumi ${ }^{(2)}$ e André Rostand Ramalho ${ }^{(4)}$
}

\begin{abstract}
Resumo - Este trabalho teve o objetivo de avaliar o desempenho, a estabilidade e a adaptabilidade de 21 cultivares de soja (Glycine max (L.) Merrill), em cinco épocas de plantio no cerrado de Rondônia. $\mathrm{O}$ delineamento experimental foi blocos ao acaso, com três repetições. Os ensaios foram conduzidos no ano de 1996/97 pelo Centro de Pesquisa Agroflorestal de Rondônia, no Campo Experimental de Vilhena em Rondônia, RO. Os métodos utilizados apresentaram concordância entre os resultados alcançados, sendo que o comportamento das cultivares nas diferentes épocas de semeadura pode ser representado por um modelo linear. $O$ coeficiente de resposta linear $\beta_{1 \mathrm{i}}$ não apresentou diferença significativa $(\mathrm{P}<0,05)$ da unidade pelo teste $\mathrm{t}\left(\beta_{\mathrm{li}}=1\right)$ em todas as cultivares testadas. As cultivares que apresentaram desvios de regressão $\sigma^{2}\left(\mathrm{~d}_{\mathrm{i}}\right)$ significativos $(\mathrm{P}<0,05)$ foram MT/BR-50 e EMBRAPA 31 , com $\mathrm{R}^{2}=58,56 \%$ e $74,43 \%$, respectivamente. As demais cultivares não apresentaram desvios de regressão significativos.
\end{abstract}

Termos para indexação: Glycine max, interação genótipo-ambiente, métodos de melhoramento.

\section{Adaptability and stability of soybean cultivars in five planting dates in the Rondonian Cerrado, Brazil}

\begin{abstract}
The objetive of this study was to evaluate the stability and adaptability of soybean (Glycine $\max (\mathrm{L}$.) Merrill) cultivars in five different planting dates. The tests were composed by 21 cultivars outlined in a random blocks design, with three replicates, conduced in 1996/97 by Embrapa-Centro de Pesquisa Agroflorestal de Rondônia, in a field of Vilhena, RO, Brazil. The used methods showed accordance between the achieved results, being the behavior of cultivars in different times of sowing represented by a linear model. The coefficient of linear regression $\beta_{\mathrm{li}}$ did not show any significant difference of the unit by the $t$ test for all the tested cultivars $(\mathrm{P}<0.05)$. The cultivars which presented significant regression deviation $\sigma^{2}\left(\mathrm{~d}_{\mathrm{i}}\right)(\mathrm{P}<0.05)$ were MT/BR-50 e EMBRAPA $31, \mathrm{R}^{2}=58.56 \%$ e $74.43 \%$, respectively. The other cultivars did not present any significant regression deviation.
\end{abstract}

Index terms: Glycine max, genotype environment interaction, breeding methods.

\section{Introdução}

A cultura da soja no estado de Rondônia tem se concentrado na região sul do Estado (Chapada dos Parecis), caracterizada por clima do tipo Aw, segun-

(1) Aceito para publicação em 14 de julho de 2000

(2)Embrapa-Centro de Pesquisa Agroflorestal de Rondônia (CPAF-RO), Caixa Postal 405, CEP 78995-000, Vilhena, RO. E-mail: prado@netview.com.br, vgodinho@netview.com.br, marleyutumi@netview.com.br

(3) Embrapa-Centro Nacional de Pesquisa de Soja (CNPSo)/ Fundação Mato Grosso, Caixa Postal 79, CEP 78705-040 Rondonópolis, MT. E-mail: fundacaomt@fundacaomt.com.br

(4)Embrapa-CPAF-RO, Caixa Postal 406, CEP 78900-970 Porto Velho, RO. E-mail: rostand@netview.com.br do a classificação de Köppen. A precipitação média anual é de $2.000 \mathrm{~mm}$, concentrada nos meses de novembro a março, com temperatura média de $24,6^{\circ} \mathrm{C}$ e umidade relativa do ar média de $74 \%$, com estação seca bem definida e altitude variando de 600 a $630 \mathrm{~m}$. O solo predominante é classificado como Latossolo Vermelho-Amarelo distrófico, fase cerrado, relevo plano.

Com a implementação do corredor de exportação constituído pela BR 364, Porto Velho, Hidrovia Madeira-Amazonas, criou-se a perspectiva da expansão da cultura da soja em direção à Região Norte. Diante deste novo cenário, experimentos que investiguem épocas adequadas de plantio e o comportamento de cultivares em face das condições ambientais 
assumem um caráter primordial para indicação de cultivares.

Para o caráter rendimento de grãos, o fenótipo $(\mathrm{F})$ a ser obtido depende do genótipo (G), do ambiente (A) e da interação dos genótipos por ambiente (GA). Este último componente ocorre porque o desempenho dos genótipos não é consistente nos vários ambientes e reflete as diferentes respostas dos genótipos às mudanças ambientais.

Considerando as inúmeras variações ambientais a que a soja é comumente submetida no Brasil, é esperado que a interação genótipo por ambiente assuma papel fundamental na manifestação fenotípica, devendo, portanto, ser estimada e considerada na indicação de cultivares e no programa de melhoramento genético.

Diversos métodos têm sido propostos para estudo da estabilidade: o método tradicional de Plaisted \& Peterson (1959), Finlay \& Wilkinson (1963), Wricke (1965), Eberhart \& Russell (1966), Perkins \& Jinks (1968), Freeman \& Perkins (1971), Tai (1971), Verma et al. (1978), Silva \& Barreto (1986) e Cruz et al. (1989). A diferença entre os métodos origina-se nos próprios conceitos da estabilidade e nos procedimentos biométricos empregados para medi-la.

Pelo modelo de regressão linear segmentada segundo Silva \& Barreto (1986), a adaptabilidade de um genótipo é caracterizada pelos seguintes parâmetros: rendimento médio $\left(\beta_{0 \mathrm{i}}+\beta_{2 \mathrm{i}} \mathrm{x}_{2}\right.$, onde $\mathrm{x}_{2}$. é a média dos índices de ambiente positivos), taxa de resposta nos ambientes desfavoráveis $\left(\beta_{1 i}\right)$ e taxa de resposta nos ambientes favoráveis $\left(\beta_{1 \mathrm{i}}+\beta_{2 \mathrm{i}}\right)$. O genótipo desejável é aquele que possua elevado rendimento médio, taxa de resposta baixa nos ambientes desfavoráveis, e elevada nos ambientes favoráveis. Segundo Eberhart \& Russell (1966), a cultivar ideal é aquela que tem produção média alta, coeficiente de regressão igual à unidade e desvio de regressão pequeno, isto é, aquele desvio que tem resposta positiva à melhoria das condições ambientais $\left(\beta_{1}=1,0\right)$, e comportamento altamente previsível $\left(\sigma^{2} \mathrm{~d}_{\mathrm{i}}=0,0\right)$.

Easton \& Clements (1973) e Lin et al. (1986) salientaram que a caracterização da adaptabilidade e estabilidade fenotípica é relativa ao conjunto dos genótipos incluídos no experimento. Como a produtividade do ambiente é expressa pelo próprio desem- penho médio dos genótipos no experimento, não há qualquer segurança de que um genótipo manterá o mesmo comportamento quando avaliado com diferentes genótipos.

Lin et al. (1986) apresentaram críticas aos métodos de avaliar a estabilidade baseada no desvio da regressão. Segundo os autores, o desvio da regressão serve para indicar o ajuste dos dados à equação obtida, ao invés de maior ou menor estabilidade da cultivar. Reiteram que baixa adaptação representada por $\sigma_{\text {di }}^{2}$ grande ou coeficiente de determinação $\left(\mathrm{R}_{\mathrm{i}}{ }^{2}\right)$ pequeno deve ser interpretado como indicativo de que o uso do modelo de regressão para estimar a estabilidade não é apropriado e que outras alternativas devam ser investigadas.

Sakiyama et al. (1988), analisando 46 ensaios de avaliação de genótipos de soja, em 16 locais de Minas Gerais, por dois anos consecutivos, fizeram comparações com a seleção realizada em apenas duas localidades em três épocas de plantio. Os autores concluíram que esta última alternativa pode ser muito eficiente se a escolha dos locais for adequada.

Segundo Ramalho et al. (1993), quando o comportamento de duas cultivares são concordantes em dois ambientes distintos, a interação é chamada de interação simples, não acarretando maiores problemas. Entretanto, quando as cultivares possuem comportamento diverso, a interação é denominada complexa. Considerando um número maior de ambientes e de cultivares, a presença de interação complexa quase sempre indica a existência de cultivares especificamente adaptadas a ambientes particulares, bem como de outras com adaptação mais ampla, porém nem sempre com alto potencial produtivo. Estes autores apresentaram três maneiras de atenuar os efeitos da interação genótipos versus ambientes: 1) identificar cultivares específicas para cada ambiente, 2) zoneamento ecológico e 3 ) identificação de cultivares com maior estabilidade fenotípica.

Alliprandini et al. (1994) analisaram os efeitos da interação genótipo $\mathrm{x}$ ambiente sobre a produtividade da soja, em vários locais e por vários anos, no Estado do Paraná, e concluíram que o componente de ano x local foi o de maior importância para as condições do experimento, correspondendo a 58,5\%, $57,0 \%$ e $37,1 \%$ da variabilidade ambiental dos grupos L (ciclo precoce), M (ciclo semi-precoce), e 
$\mathrm{N}$ (ciclo médio), respectivamente. Outros componentes significativos corresponderam aos efeitos de ano $\mathrm{x}$ local $\mathrm{x}$ genótipo nos grupos $\mathrm{L}, \mathrm{M}$, e $\mathrm{Ne}$, local $\mathrm{x}$ genótipo no grupo N. Os autores concluíram, ainda, que, no caso em que a recomendação de cultivares é necessária com apenas um ano de teste de produtividade, seria conveniente que este teste fosse realizado em vários locais.

O objetivo deste trabalho foi avaliar o desempenho, a estabilidade e a adaptabilidade de 21 cultivares de soja, em cinco épocas de plantio, no cerrado de Rondônia, através dos métodos de Wricke (1965), Cruz et al. (1989) e Eberhart \& Russell (1966).

\section{Material e Métodos}

No ano agrícola de 1996/97, foram avaliadas as seguintes cultivares de soja: FT-Estrela; MGBR-46 (Conquista); MT/BR-49 (Pioneira); MT/BR-45 (Paiaguás); MT/ BR-51 (Xingu); MT/BR-50 (Parecis); IAC-8; MT/BR-52 (Curió); EMBRAPA 9 (Bays); EMBRAPA 30 (Vale do Rio Doce); EMBRAPA 28 (Seridó); MT/BR-53 (Tucano); EMBRAPA 31 (Mina); Milionária; FT-Cristalina (Cristalina); MT/BR-47 (Canário); EMBRAPA 20 (Doko RC); EMGOPA-308 (Serra Dourada); BR/EMGOPA-314 (Garça Branca); EMGOPA-313 Rch (Jataí) e MT/BR-55 (Uirapuru), em cinco épocas de plantio: 5/11/96; 15/11/96, 30/11/96, 15/12/96, 1/1/97. A unidade experimental constituiu-se de quatro linhas de $5 \mathrm{~m}$ com espaços, entre si, de $0,45 \mathrm{~m}$ e densidade de plantio de 400.000 plantas/ha. Como área útil, utilizaram-se $3,6 \mathrm{~m}^{2}$, ou seja, as duas linhas centrais, retirando-se $0,50 \mathrm{~m}$ de cada extremidade. Foi utilizado o delineamento em blocos ao acaso, com 21 tratamentos e três repetições por época de plantio.

O ensaio foi instalado no Campo Experimental de Vilhena, da Embrapa-Centro de Pesquisa Agroflorestal de Rondônia (1245' S e $60^{\circ} 08^{\prime} \mathrm{W}, 600 \mathrm{~m}$ de altitude), sob o domínio do ecossistema de Cerrado, relevo plano, Latossolo Vermelho-Amarelo distrófico, com clima local do tipo Aw, segundo a classificação de Köppen. A precipitação média anual é de $2.000 \mathrm{~mm}$, concentrada nos meses de novembro a março, com temperatura média de $24,6^{\circ} \mathrm{C}$ e umidade relativa do ar de $74 \%$, com estação seca bem definida nos meses de maio a setembro.

A correção do solo foi feita com 6,0 t/ha de calcário dolomítico (PRNT $=65 \%$ ), e dividida em duas aplicações. Posteriormente, a área experimental foi cultivada com arroz, seguido de dois anos com soja. As características químicas do solo, antes da instalação do experimento, eram:
$\mathrm{pH} \mathrm{em} \mathrm{CaCl}_{2} 5,6 ; 5,4 \mathrm{cmol}_{\mathrm{c}} / \mathrm{dm}^{3} \mathrm{de} \mathrm{P} ; 0,07 \mathrm{cmol}_{\mathrm{c}} / \mathrm{dm}^{3} \mathrm{de}$ $\mathrm{K} ; 3,0 \mathrm{cmol}_{\mathrm{c}} / \mathrm{dm}^{3}$ de $\mathrm{Ca} ; 1,0 \mathrm{cmol}_{\mathrm{c}} / \mathrm{dm}^{3}$ de $\mathrm{Mg}$; $0,0 \mathrm{cmol}_{\mathrm{c}} / \mathrm{dm}^{3} \mathrm{de} \mathrm{Al} ; 3,0 \mathrm{cmol}_{\mathrm{c}} / \mathrm{dm}^{3} \mathrm{de} \mathrm{H} ; \mathrm{e}, 3,0 \mathrm{dag} / \mathrm{kg} \mathrm{de}$ M.O. A adubação no sulco de plantio foi de $100 \mathrm{~kg} / \mathrm{ha} \mathrm{de}$ $\mathrm{P}_{2} \mathrm{O}_{5}, 72 \mathrm{~kg} / \mathrm{ha} \mathrm{de} \mathrm{K}_{2} \mathrm{O}$ e $40 \mathrm{~kg} / \mathrm{ha} \mathrm{de} \mathrm{FTE} \mathrm{Cerrado.}$

Os dados correspondentes a rendimento de grãos $(\mathrm{kg} / \mathrm{ha})$, altura de plantas (cm) e altura de inserção da primeira vagem $(\mathrm{cm})$ foram avaliados individualmente e de maneira conjunta, nas cinco épocas de plantio em fatorial simples, com desdobramento da soma de quadrados em efeitos de épocas, de genótipos e da interação épocas $\mathrm{x}$ genótipos.

A análise de estabilidade e adaptabilidade das cultivares, relativa ao rendimento de grãos $(\mathrm{kg} / \mathrm{ha})$, foi feita por três métodos distintos: pelo método da ecovalência, proposto por Wricke (1965), pelo método bissegmentado da regressão (Cruz et al., 1989), e pelo método da regressão linear proposto por Eberhart \& Russell (1966). Quanto à altura da planta e altura da inserção da primeira vagem foram apenas estimados os coeficientes $\mathrm{W}_{\mathrm{i}} \%$.

Os modelos matemáticos para os métodos de Cruz et al. (1989) e Eberhart \& Russell (1966) são semelhantes; a diferença é que para o modelo de Cruz et al. (1989) foi introduzido o coeficiente de regressão nos ambientes desfavoráveis, formando dois segmentos de reta. O modelo matemático no método bissegmentado de Cruz et al. (1989) é dado por: $\mathrm{Y}_{\mathrm{ij}}=\beta_{0 \mathrm{i}}+\beta_{1 \mathrm{i}} \mathrm{I}_{\mathrm{j}}+\beta_{2 \mathrm{i}} \mathrm{T}\left(\mathrm{I}_{\mathrm{j}}\right)+\delta_{\mathrm{ij}}+\mathrm{c}_{\mathrm{ij}}$. em que: $\beta_{0 \mathrm{i}}=$ média geral do genótipo i $(\mathrm{i}=1,2, \ldots, \mathrm{g}) ; \beta_{1 \mathrm{i}}=$ resposta linear do genótipo i à variação ambiental; $\mathrm{I}_{\mathrm{j}}=$ índice ambiental $(\mathrm{j}=1,2, \ldots, \mathrm{a})$, sendo $\mathrm{Ij}=(\mathrm{Y} . \mathrm{j} / \mathrm{g})-(\mathrm{Y} . . / \mathrm{ga}) ; \delta_{\mathrm{ij}}=\mathrm{des}-$ vio da regressão; $c_{i j}=$ erro experimental médio; $T\left(\mathrm{I}_{\mathrm{j}}\right)=0$ se $\mathrm{I}_{\mathrm{j}}<0 ; \mathrm{T}\left(\mathrm{I}_{\mathrm{j}}\right)=\mathrm{I}_{\mathrm{j}}-\hat{\mathrm{I}}_{+}$se $\mathrm{I}_{\mathrm{j}}>0$, sendo $\hat{\mathrm{I}}_{+}$a média dos índices $\mathrm{I}_{\mathrm{j}}$ positivos. Semelhantemente, o modelo de Eberhart \& Russell (1966) é dado por $Y_{\mathrm{ij}}=\beta_{0 \mathrm{i}}+\beta_{1 \mathrm{i}} \mathrm{I}_{\mathrm{j}}+\delta_{\mathrm{ij}}+c_{\mathrm{ijj}}$.

As hipóteses $\left(\mathrm{H}_{0}: \beta_{1 \mathrm{i}}=1\right),\left(\mathrm{H}_{0}: \beta_{2 \mathrm{i}}=0\right)$ e $\left(\mathrm{H}_{0}:\left(\beta_{1 \mathrm{i}}+\beta_{2 \mathrm{i}}\right)=1\right)$ foram testadas pelo teste " $\mathrm{t}_{\alpha, \mathrm{m}}$ ", sendo $\alpha$ o nível de significância, e m, os graus de liberdade do resíduo. Os testes foram obtidos pelas fórmulas: $\mathrm{t}=\left(\beta_{1 \mathrm{i}}-1\right)$ $\left(\sigma^{2} \beta_{1 \mathrm{i}}\right)^{1 / 2} ; \mathrm{t}=\left(\beta_{2 \mathrm{i}}\right) /\left(\sigma^{2} \beta_{2 \mathrm{i}}\right)^{1 / 2} ; \mathrm{t}=\left(\beta_{1 \mathrm{i}}+\beta_{2 \mathrm{i}}\right)-1 /\left(\sigma^{2} \beta_{1 \mathrm{i}}+\beta_{2 \mathrm{i}}\right)^{1 / 2}$, respectivamente.

Para a análise dos dados, foi utilizado o aplicativo computacional em genética e estatística Genes (Cruz, 1997).

\section{Resultados e Discussão}

As médias obtidas, assim como a análise de variância, a estimativa da variância genética e a herdabilidade do rendimento de grãos ( $\mathrm{kg} / \mathrm{ha}$ ), altura das plantas $(\mathrm{cm})$ e altura da inserção da primeira vagem $(\mathrm{cm})$ das 21 cultivares de soja cultivadas em cinco épocas de plantio, são apresentadas nas 
Tabelas 1, 2 e 3 . O rendimento médio de grãos da primeira à quinta época de plantio variaram de 2.316,70 a $3.165,09 \mathrm{~kg} / \mathrm{ha}$.

A média geral, nos cinco ambientes avaliados, foi de $2.743,06 \mathrm{~kg} / \mathrm{ha}$ quanto a rendimento de grãos, $70,66 \mathrm{~cm}$ quanto à altura das plantas e $21,61 \mathrm{~cm}$ quanto à altura de inserção da primeira vagem. Os coeficientes de variação experimental foram baixos, variando apenas de $6,11 \%$ a $14,27 \%$, o que evidencia a precisão dos dados experimentais.

A análise de variância conjunta, relativa a rendimento de grãos $(\mathrm{kg} / \mathrm{ha})$, altura das plantas $(\mathrm{cm})$ e altura da inserção da primeira vagem $(\mathrm{cm})$, encontrase na Tabela 4. A análise de variância apresentou efeitos significativos $(\mathrm{P}<0,01)$ de genótipos, de ambientes, e da interação genótipos $\mathrm{x}$ ambientes referente a altura das plantas e altura da inserção da primeira vagem. No tocante a rendimento de grãos, o efeito da interação genótipos x ambiente não foi significativo.

Os resultados de estabilidade e adaptabilidade das cultivares de soja, quanto ao rendimento de grãos $(\mathrm{kg} / \mathrm{ha})$, à altura de plantas $(\mathrm{cm})$ e à altura de inserção da primeira vagem $(\mathrm{cm})$, utilizando-se o método da ecovalência proposto por Wricke (1965), encontramse na Tabela 5. A análise da estabilidade e adaptabilidade do rendimento de grãos ao longo das cinco épocas de plantio pelos métodos da regressão

Tabela 1. Produtividade média de grãos (kg/ha) de 21 cultivares de soja semeadas em cinco épocas de plantio. Vilhena, RO.

\begin{tabular}{|c|c|c|c|c|c|c|}
\hline Cultivar & $5 / 11 / 96$ & $15 / 11 / 96$ & $30 / 11 / 96$ & $15 / 12 / 96$ & 1/1/97 & Média \\
\hline MT/BR-55 & $3.789,26$ & $3.521,95$ & $3.137,40$ & $3.076,43$ & $2.729,40$ & $3.250,89$ \\
\hline MT/BR-53 & $3.385,95$ & $3.231,19$ & $3.123,33$ & $2.696,57$ & $2.813,81$ & $3.050,17$ \\
\hline MT/BR-50 & $3.306,23$ & $3.306,23$ & $3.545,40$ & $2.738,77$ & $2.297,94$ & $3.038,91$ \\
\hline MT/BR-51 & $3.395,33$ & $3.259,33$ & $3.057,68$ & $2.832,57$ & $2.630,91$ & $3.035,16$ \\
\hline BR/EMGOPA-314 & $3.526,64$ & $2.945,12$ & $3.010,78$ & $2.673,12$ & $2.527,74$ & $2.936,68$ \\
\hline FT-Cristalina & $3.278,09$ & $3.109,26$ & $2.729,40$ & $3.062,36$ & $2.335,46$ & $2.902,91$ \\
\hline EMGOPA-308 & $3.287,47$ & $2.968,57$ & $2.949,81$ & $2.724,71$ & $2.579,32$ & $2.901,98$ \\
\hline MT/BR-52 & $2.977,95$ & $3.409,40$ & $2.851,33$ & $2.644,98$ & $2.269,81$ & $2.830,69$ \\
\hline EMGOPA-313 Rch & $3.409,40$ & $3.057,67$ & $2.818,50$ & $2.565,26$ & $2.115,05$ & $2.793,18$ \\
\hline MT/BR-49 & $3.273,40$ & $2.691,88$ & $2.630,91$ & $2.555,88$ & $2.602,77$ & $2.750,97$ \\
\hline MT/BR-47 & $3.409,40$ & $3.057,68$ & $2.537,12$ & $2.584,02$ & $2.166,63$ & $2.750,97$ \\
\hline IAC-8 & $3.057,67$ & $3.357,81$ & $2.626,22$ & $2.166,63$ & $2.330,77$ & $2.707,82$ \\
\hline EMBRAPA 30 & $3.001,40$ & $2.949,81$ & $2.340,15$ & $2.649,67$ & $2.541,81$ & $2.696,57$ \\
\hline MT/BR-45 & $3.048,29$ & $3.113,95$ & $2.480,84$ & $2.391,74$ & $2.302,63$ & $2.667,49$ \\
\hline FT-Estrela & $3.038,91$ & $3.113,95$ & $2.523,05$ & $2.335,46$ & $2.246,36$ & $2.651,55$ \\
\hline EMBRAPA 31 & $2.959,19$ & $3.235,88$ & $2.977,95$ & $2.105,67$ & $1.964,98$ & $2.648,73$ \\
\hline MGBR-46 & $3.287,47$ & $2.870,09$ & $2.377,67$ & $2.326,08$ & $2.340,15$ & $2.640,29$ \\
\hline EMBRAPA 20 & $3.128,02$ & $2.584,02$ & $2.269,81$ & $2.607,46$ & $2.007,18$ & $2.519,30$ \\
\hline EMBRAPA 28 & $2.738,77$ & $2.668,43$ & $2.776,29$ & $2.166,63$ & $2.119,74$ & $2.493,97$ \\
\hline EMBRAPA 9 & $2.630,91$ & $2.659,05$ & $2.241,67$ & $2.124,43$ & $2.138,50$ & $2.358,91$ \\
\hline Milionária & $2.537,12$ & $2.321,39$ & $1.580,42$ & $1.857,11$ & $1.589,80$ & $1.977,17$ \\
\hline Q.M.(Bloco) (2 g.1.) & $427.693,34$ & $419.700,91$ & $239.583,44$ & $84.514,51$ & $74.807,34$ & \\
\hline Q.M.(Trat) (20 g.l.) & $273.595,18$ & $277.787,04$ & $523.569,92$ & $299.810,12$ & $247.891,26$ & \\
\hline Q.M.(Res) (40 g.1.) & $130.221,94$ & $185.738,10$ & $116.974,35$ & $124.794,08$ & $55.456,54$ & \\
\hline $\mathrm{F}$ & $2,10 *$ & $1,50^{\mathrm{ns}}$ & $4,47 * *$ & $2,40 * *$ & $4,47 * *$ & \\
\hline Média & $3.165,09$ & $3.020,60$ & $2.694,56$ & $2.518,36$ & $2.316,70$ & $2.743,06$ \\
\hline $\mathrm{CV}(\%)$ & 11,40 & 14,27 & 12,69 & 14,03 & 10,17 & 12,77 \\
\hline$\sigma_{g}^{2}$ & $47.791,08$ & $30.682,98$ & $135.531,86$ & $58.338,68$ & $64.144,91$ & $67.168,73$ \\
\hline$h^{2}$ & 0,52 & 0,33 & 0,78 & 0,58 & 0,78 & 89,15 \\
\hline
\end{tabular}

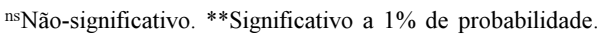


bissegmentada encontra-se nas Tabelas 6 a 9, e o da regressão linear de Eberhart \& Russell (1966), nas Tabelas 10 e 11.

Segundo o método da ecovalência $\left(\mathrm{W}_{\mathrm{i}}{ }^{2}\right)$, o material genético mais estável é o que apresenta menor estimativa para a ecovalência, ou seja, o genótipo com menor contribuição para a interação genótipo $\mathrm{x}$ ambiente. Utilizando este método e os dados de rendimento de grãos, as cultivares foram ordenadas em ordem decrescente de estabilidade: MT/BR-51, MT/BR-55, FT-Estrela, MT/BR-45, EMGOPA-308, EMBRAPA 9, MT/BR-53, EMGOPA-313 Rch, MT/BR-47, BR/EMGOPA-314, MGBR-46, Milionária,
EMBRAPA 28, FT-Cristalina, MT/BR-52, MT/BR-49, EMBRAPA 30, EMBRAPA 20, IAC-8, EMBRAPA 31 e MT/BR-50. Estes dados fornecem indicativos para a recomendação de cultivares que acompanham o desempenho médio obtido nos ambientes, e portanto, capazes de apresentar os melhores desempenhos em várias épocas de plantio. As cultivares MT/BR-50 e EMBRAPA 31 apresentaram os maiores valores de $\left(\mathrm{W}_{\mathrm{i}} \%\right.$ ), 13,30\% e 12,75\%, respectivamente (Tabela 5), demonstrando, assim, a grande interação que possuem com o ambiente, sendo que a cultivar MT/BR-50 apresentou desempenho médio $(3.038,91 \mathrm{~kg} / \mathrm{ha}) \mathrm{su}$ perior a EMBRAPA $31(2.648,73 \mathrm{~kg} / \mathrm{ha})$ (Tabela 1).

Tabela 2. Altura média das plantas $(\mathrm{cm})$ de 21 cultivares de soja semeadas em cinco épocas de plantio. Vilhena, RO.

\begin{tabular}{|c|c|c|c|c|c|c|}
\hline Cultivar & $5 / 11 / 96$ & $15 / 11 / 96$ & $30 / 11 / 96$ & $15 / 12 / 96$ & $1 / 1 / 97$ & Média \\
\hline MT/BR-55 & 73,67 & 75,00 & 61,00 & 60,00 & 65,00 & 66,93 \\
\hline MT/BR-53 & 71,67 & 73,33 & 66,67 & 53,33 & 63,00 & 65,60 \\
\hline MT/BR-50 & 62,33 & 68,00 & 61,33 & 55,00 & 62,33 & 61,80 \\
\hline MT/BR-51 & 51,00 & 57,67 & 52,33 & 44,67 & 57,33 & 52,60 \\
\hline BR/EMGOPA-314 & 72,00 & 71,00 & 66,00 & 63,67 & 68,67 & 68,27 \\
\hline FT-Cristalina & 61,00 & 63,00 & 60,33 & 50,33 & 51,00 & 57,13 \\
\hline EMGOPA-308 & 76,33 & 77,33 & 73,00 & 73,67 & 68,67 & 73,80 \\
\hline MT/BR-52 & 78,00 & 81,33 & 69,33 & 66,33 & 66,33 & 72,27 \\
\hline EMGOPA-313 Rch & 54,33 & 65,33 & 66,00 & 57,67 & 66,00 & 61,87 \\
\hline MT/BR-49 & 65,00 & 72,00 & 68,33 & 59,33 & 66,67 & 66,27 \\
\hline MT/BR-47 & 63,67 & 69,67 & 64,67 & 56,33 & 69,67 & 64,80 \\
\hline IAC-8 & 79,33 & 87,33 & 81,00 & 76,00 & 81,67 & 81,07 \\
\hline EMBRAPA 30 & 94,00 & 101,00 & 85,67 & 86,00 & 87,33 & 90,80 \\
\hline MT/BR-45 & 82,67 & 78,67 & 79,33 & 67,33 & 76,67 & 76,93 \\
\hline FT-Estrela & 46,00 & 50,67 & 51,33 & 40,00 & 54,67 & 48,53 \\
\hline EMBRAPA 31 & 84,67 & 86,00 & 74,67 & 60,67 & 64,33 & 74,07 \\
\hline MGBR-46 & 62,67 & 63,33 & 67,67 & 62,00 & 64,67 & 64,07 \\
\hline EMBRAPA 20 & 71,33 & 72,00 & 68,67 & 60,00 & 64,00 & 67,20 \\
\hline EMBRAPA 28 & 100,67 & 95,33 & 66,33 & 80,00 & 80,00 & 84,47 \\
\hline EMBRAPA 9 & 92,00 & 85,33 & 78,33 & 67,00 & 77,33 & 80,00 \\
\hline Milionária & 104,67 & 109,67 & 107,33 & 101,00 & 104,00 & 105,33 \\
\hline Q.M.(Bloco) (2 g.l.) & 62,90 & 7,05 & 3,16 & 2,40 & 12,26 & \\
\hline Q.M.(Trat) (20 g.1.) & 742,47 & 610,97 & 442,96 & 580,59 & 422,32 & \\
\hline Q.M.(Res) (40 g.1.) & 43,92 & 22,51 & 50,36 & 15,21 & 20,02 & \\
\hline $\mathrm{F}$ & $16,90 * *$ & $27,14 * *$ & $8,80 * *$ & $38,16 * *$ & $21,09 * *$ & \\
\hline Média & 73,67 & 76,33 & 69,97 & 63,83 & 69,49 & 70,66 \\
\hline $\mathrm{CV}(\%)$ & 9,00 & 6,22 & 10,14 & 6,11 & 6,44 & 7,80 \\
\hline$\sigma_{g}^{2}$ & 232,85 & 196,15 & 130,87 & 188,46 & 134,10 & \\
\hline$h^{2}$ & 0,94 & 0,96 & 0,89 & 0,97 & 0,95 & \\
\hline
\end{tabular}

**Significativo a $1 \%$ de probabilidade. 
As cultivares com menor contribuição para a interação genótipo x ambiente foram: MT/BR-51 $\left(\mathrm{W}_{\mathrm{i}} \%=0,39 \%\right)$ e MT/BR-55 $\left(\mathrm{W}_{\mathrm{i}} \%=0,90 \%\right)$, portanto, segundo o conceito da ecovalência, as de maior estabilidade. Estes resultados concordam com os obtidos pelo método de Eberhart \& Russell (1966) (Tabela 11).

As estimativas de ecovalência para o rendimento de grãos, altura das plantas e altura da inserção da primeira vagem dentro das cultivares apresentaram diferentes valores de $\mathrm{W}_{\mathrm{i}} \%$, o que indica que o material mais estável para rendimento de grãos não é necessariamente o mais estável para altura das plantas ou para a altura da inserção da primeira vagem ao longo das épocas de plantio.

Os maiores valores de $\mathrm{W}_{\mathrm{i}} \%$ referentes a altura das plantas foram encontrados nas cultivares EMBRAPA 28, EMBRAPA 31 e EMGOPA-313 Rch.

Tabela 3. Altura média de inserção da primeira vagem $(\mathrm{cm})$ de 21 cultivares de soja semeadas em cinco épocas de plantio. Vilhena, RO.

\begin{tabular}{|c|c|c|c|c|c|c|}
\hline Cultivar & $5 / 11 / 96$ & $15 / 11 / 96$ & $30 / 11 / 96$ & $15 / 12 / 96$ & $1 / 1 / 97$ & Média \\
\hline MT/BR-55 & 21,67 & 24,67 & 22,33 & 20,33 & 24,33 & 22,67 \\
\hline MT/BR-53 & 19,33 & 20,67 & 21,00 & 17,33 & 20,00 & 19,67 \\
\hline MT/BR-50 & 21,67 & 21,00 & 15,67 & 17,67 & 21,67 & 19,53 \\
\hline MT/BR-51 & 16,33 & 18,33 & 15,33 & 14,00 & 16,33 & 16,07 \\
\hline BR/EMGOPA-314 & 19,00 & 20,00 & 17,67 & 18,67 & 19,67 & 19,00 \\
\hline FT-Cristalina & 15,67 & 19,00 & 18,00 & 16,33 & 18,33 & 17,47 \\
\hline EMGOPA-308 & 20,00 & 19,67 & 20,00 & 19,33 & 21,67 & 20,13 \\
\hline MT/BR-52 & 20,33 & 23,33 & 20,00 & 20,00 & 24,33 & 21,60 \\
\hline EMGOPA-313 Rch & 13,00 & 18,33 & 14,33 & 15,33 & 19,33 & 16,07 \\
\hline MT/BR-49 & 22,67 & 26,67 & 23,00 & 22,33 & 24,00 & 23,73 \\
\hline MT/BR-47 & 19,00 & 19,00 & 20,67 & 19,00 & 23,67 & 20,27 \\
\hline IAC- 8 & 25,00 & 26,00 & 18,33 & 21,33 & 20,00 & 22,13 \\
\hline EMBRAPA 30 & 19,67 & 20,67 & 23,00 & 21,67 & 20,67 & 21,13 \\
\hline MT/BR-45 & 21,67 & 26,33 & 23,67 & 21,33 & 26,00 & 23,80 \\
\hline FT-Estrela & 13,33 & 17,00 & 16,33 & 10,00 & 17,67 & 14,87 \\
\hline EMBRAPA 31 & 20,33 & 22,33 & 21,67 & 17,67 & 20,67 & 20,53 \\
\hline MGBR-46 & 21,00 & 22,33 & 20,00 & 23,67 & 24,67 & 22,33 \\
\hline EMBRAPA 20 & 23,67 & 25,67 & 23,00 & 21,00 & 26,67 & 24,00 \\
\hline EMBRAPA 28 & 25,00 & 23,67 & 24,00 & 31,33 & 25,33 & 25,87 \\
\hline EMBRAPA 9 & 27,00 & 24,67 & 25,67 & 23,00 & 24,33 & 24,93 \\
\hline Milionária & 35,67 & 32,67 & 42,00 & 37,33 & 42,33 & 38,00 \\
\hline Q.M.(Bloco) (2 g.l.) & 5,90 & 9,90 & 8,44 & 12,97 & 3,92 & \\
\hline Q.M.(Trat) (20 g.1.) & 72,27 & 42,28 & 97,38 & 97,96 & 84,42 & \\
\hline Q.M.(Res) (40 g.1.) & 3,72 & 5,50 & 4,01 & 6,65 & 3,59 & \\
\hline $\mathrm{F}$ & $19,42 * *$ & $7,68 * *$ & $24,28 * *$ & $14,73 * *$ & $23,53 * *$ & \\
\hline Média & 21,00 & 22,48 & 21,22 & 20,41 & 22,94 & 21,61 \\
\hline $\mathrm{CV}(\%)$ & 9,19 & 10,44 & 9,44 & 12,64 & 8,26 & 10,03 \\
\hline$\sigma_{g}^{2}$ & 22,85 & 12,26 & 31,12 & 30,44 & 26,94 & \\
\hline$h^{2}$ & 0,95 & 0,87 & 0,96 & 0,93 & 0,96 & \\
\hline
\end{tabular}

**Significativo a $1 \%$ de probabilidade. 
Quanto à altura da inserção da primeira vagem, os maiores valores para $\mathrm{Wi} \%$ foram encontrados nas cultivares Milionária, EMBRAPA 28, IAC-8 e FT-Estrela.

Tabela 4. Análise de variância conjunta para rendimento de grãos $(\mathrm{kg} / \mathrm{ha})$, altura das plantas $(\mathrm{cm})$ e altura da inserção da primeira vagem $(\mathrm{cm})$ de 21 cultivares de soja semeadas em cinco épocas de plantio. Vilhena, RO.

\begin{tabular}{|c|c|c|c|c|}
\hline \multirow{2}{*}{$\begin{array}{l}\text { Fonte de } \\
\text { variação }\end{array}$} & \multirow[t]{2}{*}{ GL } & \multicolumn{3}{|c|}{ Quadrado médio } \\
\hline & & $\begin{array}{l}\text { Procedimentos } \\
\text { de grãos }\end{array}$ & $\begin{array}{c}\text { Altura } \\
\text { de plantas }\end{array}$ & $\begin{array}{c}\text { Inserção } \\
\text { da } 1^{\mathrm{a}} \\
\text { vagem } \\
\end{array}$ \\
\hline Blocos/Amb. & 10 & $249.258,81$ & 17,55 & 8,23 \\
\hline Genótipos & 20 & $1.130 .167,99 * *$ & $2.524,70^{* *} *$ & $344,32 *$ \\
\hline Ambientes & 4 & $7.7137 .38,75^{* *}$ & $1.414,06^{* *}$ & $70,33 *$ \\
\hline Gen.xAmbientes & 80 & $123.121,25^{\mathrm{ns}}$ & $68,65 * *$ & $12,50 *$ \\
\hline Resíduo & 200 & $122.637,06$ & 30,40 & 4,70 \\
\hline Média & & $2.743,06$ & 70,66 & 21,61 \\
\hline $\mathrm{CV}(\%)$ & & 12,77 & 7,80 & 10,03 \\
\hline
\end{tabular}

${ }^{n s}$ Não-significativo. ${ }^{* *}$ Significativo a $1 \%$ de probabilidade
Na Tabela 6, estão apresentadas as médias de produtividade $(\mathrm{kg} / \mathrm{ha})$ relativas às cinco épocas de plantio, e os índices ambientais $\left(\mathrm{I}_{\mathrm{j}}\right)$ e $\mathrm{T}\left(\mathrm{I}_{\mathrm{j}}\right)$ segundo o método de Cruz et al. (1989). A análise dos dados indicou índices ambientais positivos referentes à primeira e segunda época de plantio (5/11 e 15/11), para produtividades médias acima de $2.700 \mathrm{~kg} / \mathrm{ha}$. Por este critério, as épocas 30/11, 15/12, e 1/1 foram consideradas desfavoráveis, provavelmente porque entre as cultivares testadas não existiam as que atendessem satisfatoriamente ao escalonamento de plantio em diferentes épocas de semeadura. Considerando as médias obtidas nos ambientes desfavoráveis e favoráveis (Tabela 7), as cultivares MT/BR-55, MT/BR-53, MT/BR-50, MT/BR-51, BR/EMGOPA-314, FT-Cristalina e EMGOPA-308 apresentaram rendimentos médios acima da média $(2.743,06 \mathrm{~kg} / \mathrm{ha})$, em ambas as situações.

$\mathrm{Na}$ Tabela 8 são apresentadas as estimativas de $\beta_{0}, \beta_{1 i}, \beta_{2 \mathrm{i}}$ e $\left(\beta_{1 \mathrm{i}}+\beta_{2 \mathrm{i}}\right)$, sendo desejáveis cultivares que possuam baixos valores de $\beta_{1 i}$, associados a

Tabela 5. Estimativa da ecovalência de 21 cultivares de soja semeadas em cinco épocas de plantio $(5 / 11,15 / 11,30 / 11$, $15 / 12$ e 1/1/97), quanto às características de rendimento de grãos ( $\mathrm{kg} / \mathrm{ha})$, altura das plantas ( $\mathrm{cm})$ e altura da inserção da 1a vagem $(\mathrm{cm})$, segundo o método descrito por Wricke (1965). Vilhena, RO.

\begin{tabular}{|c|c|c|c|c|c|c|c|c|c|}
\hline \multirow[t]{2}{*}{ Cultivar } & \multicolumn{3}{|c|}{ Rendimento de grãos } & \multicolumn{3}{|c|}{ Altura das plantas } & \multicolumn{3}{|c|}{ Altura da inserção da $1^{\mathrm{a}}$ vagem } \\
\hline & $\mathrm{kg} / \mathrm{ha}$ & $\left(\mathrm{W}_{\mathrm{i}}\right)$ & $\left(\mathrm{W}_{\mathrm{i}} \%\right)$ & $(\mathrm{cm})$ & $\left(\mathrm{W}_{\mathrm{i}}\right)$ & $\left(\mathrm{W}_{\mathrm{i}} \%\right)$ & $(\mathrm{cm})$ & $\left(\mathrm{W}_{\mathrm{i}}\right)$ & $\left(\mathrm{W}_{\mathrm{i}} \%\right)$ \\
\hline MT/BR-51 & $3.035,17$ & $38.089,09$ & 0,39 & 52,60 & 173,41 & 3,16 & 16,07 & 14,19 & 1,42 \\
\hline MT/BR-55 & $3.250,89$ & $88.131,60$ & 0,90 & 66,90 & 143,06 & 2,61 & 22,67 & 8,54 & 0,85 \\
\hline FT-Estrela & $2.651,55$ & $151.719,50$ & 1,54 & 48,50 & 334,83 & 6,10 & 14,87 & 64,60 & 6,46 \\
\hline MT/BR-45 & $2.667,49$ & $167.113,72$ & 1,70 & 76,90 & 122,93 & 2,24 & 23,80 & 22,62 & 2,26 \\
\hline EMGOPA-308 & $2.901,98$ & $204.357,23$ & 2,08 & 73,80 & 196,34 & 3,58 & 20,13 & 6,81 & 0,68 \\
\hline EMBRAPA 9 & $2.358,91$ & $210.759,31$ & 2,14 & 80,00 & 366,61 & 6,68 & 24,93 & 41,87 & 4,19 \\
\hline MT/BR-53 & $3.050,17$ & $252.813,50$ & 2,57 & 65,60 & 144,77 & 2,64 & 19,67 & 16,00 & 1,60 \\
\hline EMGOPA-313 Rch & $2.793,18$ & $320.195,63$ & 3,25 & 61,90 & 522,86 & 9,52 & 16,07 & 41,36 & 4,14 \\
\hline MT/BR-47 & $2.750,97$ & $337.101,84$ & 3,42 & 64,80 & 171,55 & 3,12 & 20,27 & 29,72 & 2,97 \\
\hline BR/EMGOPA-314 & $2.936,68$ & $352.382,44$ & 3,58 & 68,30 & 57,31 & 1,04 & 19,00 & 7,40 & 0,74 \\
\hline MGBR-46 & $2.640,29$ & $368.284,16$ & 3,74 & 64,10 & 314,23 & 5,72 & 22,33 & 37,43 & 3,74 \\
\hline Milionária & $1.977,17$ & $471.641,38$ & 4,79 & 105,30 & 86,46 & 1,57 & 38,00 & 209,94 & 21,00 \\
\hline EMBRAPA 28 & $2.493,97$ & $494.193,44$ & 5,02 & 84,50 & $1.565,20$ & 28,50 & 25,87 & 178,57 & 17,86 \\
\hline FT-Cristalina & $2.902,92$ & $571.117,00$ & 5,80 & 57,10 & 121,74 & 2,22 & 17,47 & 8,78 & 0,88 \\
\hline MT/BR-52 & $2.830,69$ & $571.792,25$ & 5,81 & 72,30 & 142,49 & 2,59 & 21,60 & 14,38 & 1,44 \\
\hline MT/BR-49 & $2.750,97$ & $620.314,31$ & 6,30 & 66,30 & 85,03 & 1,55 & 23,73 & 17,30 & 1,73 \\
\hline EMBRAPA 30 & $2.696,57$ & $643.548,88$ & 6,53 & 90,80 & 149,04 & 2,71 & 21,13 & 41,41 & 4,14 \\
\hline EMBRAPA 20 & $2.519,30$ & $677.349,50$ & 6,88 & 67,20 & 32,86 & 0,60 & 24,00 & 18,41 & 1,84 \\
\hline IAC-8 & $2.707,82$ & $742.856,88$ & 7,54 & 81,10 & 88,39 & 1,61 & 22,13 & 134,59 & 13,46 \\
\hline EMBRAPA 31 & $2.648,74$ & $1.255 .752,13$ & 12,75 & 74,10 & 644,96 & 11,74 & 20,53 & 22,69 & 2,27 \\
\hline MT/BR-50 & $3.038,92$ & $1.310 .179,13$ & 13,30 & 61,80 & 28,02 & 0,51 & 19,53 & 63,26 & 6,33 \\
\hline Média & $2.743,06$ & & & 70,66 & & & 21,61 & & \\
\hline
\end{tabular}


elevados valores de $\beta_{0}$ e $\left(\beta_{1 \mathrm{i}}+\beta_{2 \mathrm{i}}\right)$. Pelo teste $\mathrm{t}$ $\left(t_{5 \%, 200}=1,96\right)$, os valores obtidos para $\beta_{1 \mathrm{i}}, \beta_{2 \mathrm{i}} \mathrm{e}$ $\left(\beta_{1 i}+\beta_{2 i}\right)$, para todas as cultivares " $i$ ", não apresentaram diferença significativa da unidade nas hipóteses $\left(\mathrm{H}_{0}\right.$ : $\left.\beta_{1 \mathrm{i}}=1\right),\left(\mathrm{H}_{0}: \beta_{2 \mathrm{i}}=0\right)$ e $\left(\mathrm{H}_{0}:\left(\beta_{1 \mathrm{i}}+\beta_{2 \mathrm{i}}\right)=1\right)$. As combinações de taxa de resposta acima da média, taxa de resposta média e taxa de resposta abaixo da média, nos ambientes desfavoráveis e nos ambientes favoráveis, definem nove categorias de sensibilidade de resposta (Silva, 1995). Desta forma, os dados obtidos neste experimento, onde $\beta_{1} \cong 1$ e $\left(\beta_{1 \mathrm{i}}+\beta_{2 \mathrm{i}}\right) \cong 1$, sugerem que os genótipos envolvidos

Tabela 6. Índices ambientais $\left(\mathrm{I}_{\mathrm{j}}\right)$ e $\mathrm{T}\left(\mathrm{I}_{\mathrm{j}}\right)$ obtidos utilizando o método de Cruz et al. (1989).

\begin{tabular}{lcccc}
\hline Época & Média & Índice $\left(\mathrm{I}_{\mathrm{j}}\right)$ & Índice $\mathrm{T}\left(\mathrm{I}_{\mathrm{j}}\right)$ & Tipo \\
\hline $5 / 11 / 96$ & $3.165,09$ & 422,03 & 72,24 & $\mathrm{~F}$ \\
$15 / 11 / 96$ & $3.020,60$ & 277,54 & $-72,24$ & $\mathrm{~F}$ \\
$30 / 11 / 96$ & $2.694,56$ & $-48,51$ & 0,00 & $\mathrm{D}$ \\
$15 / 12 / 96$ & $2.518,36$ & $-224,70$ & 0,00 & $\mathrm{D}$ \\
$1 / 1 / 97$ & $2.316,70$ & $-426,36$ & 0,00 & $\mathrm{D}$ \\
\hline \multicolumn{5}{c}{349,78} \\
\hline
\end{tabular}

Tabela 7. Rendimento médio de grãos, em kg/ha, de 21 cultivares de soja em ambientes de índice negativo (desfavoráveis) e positivo (favoráveis). Vilhena, RO.

\begin{tabular}{lccc}
\hline Cultivar & Média & $\begin{array}{c}\text { Média } \\
\text { desfavorável }\end{array}$ & $\begin{array}{c}\text { Média } \\
\text { favorável }\end{array}$ \\
\hline MT/BR-55 & $3.250,89$ & $2.981,08$ & $3.655,61$ \\
MT/BR-53 & $3.050,17$ & $2.877,90$ & $3.308,57$ \\
MT/BR-50 & $3.038,91$ & $2.860,71$ & $3.306,23$ \\
MT/BR-51 & $3.035,16$ & $2.840,39$ & $3.327,33$ \\
BR/EMGOPA-314 & $2.936,68$ & $2.737,21$ & $3.235,88$ \\
FT-Cristalina & $2.902,91$ & $2.709,07$ & $3.193,68$ \\
EMGOPA-308 & $2.901,98$ & $2.751,28$ & $3.128,02$ \\
MT/BR-52 & $2.830,69$ & $2.588,70$ & $3.193,68$ \\
EMGOPA-313 Rch & $2.793,18$ & $2.499,60$ & $3.233,54$ \\
MT/BR-49 & $2.750,97$ & $2.596,52$ & $2.982,64$ \\
MT/BR-47 & $2.750,97$ & $2.429,26$ & $3.233,54$ \\
IAC-8 & $2.707,82$ & $2.374,54$ & $3.207,74$ \\
EMBRAPA 30 & $2.696,57$ & $2.510,54$ & $2.975,60$ \\
MT/BR-45 & $2.667,49$ & $2.391,74$ & $3.081,12$ \\
FT-Estrela & $2.651,55$ & $2.368,29$ & $3.076,43$ \\
EMBRAPA 31 & $2.648,73$ & $2.349,53$ & $3.097,53$ \\
MGBR-46 & $2.640,29$ & $2.347,97$ & $3.078,78$ \\
EMBRAPA 20 & $2.519,30$ & $2.294,82$ & $2.856,02$ \\
EMBRAPA 28 & $2.493,97$ & $2.354,22$ & $2.703,60$ \\
EMBRAPA 9 & $2.358,91$ & $2.168,20$ & $2.644,98$ \\
Milionária & $1.977,17$ & $1.675,78$ & $2.429,26$ \\
\hline Média geral & $2.743,06$ & $2.509,87$ & $3.092,85$ \\
\hline
\end{tabular}

na análise possuem iguais taxas de resposta nos ambientes desfavoráveis e favoráveis. Entretanto, como $\beta_{2 \mathrm{i}} \cong 0$ para todo $\mathrm{i}=1,2, \ldots, \mathrm{g}$, a confirmação da hipótese $\left(\mathrm{H}_{0}: \beta_{2 \mathrm{i}}=0\right)$ pelo teste $\mathrm{t}$ indica que a relação entre rendimento e índice de ambiente pode ser apropriadamente representada por um modelo de regressão linear simples, como o modelo de Eberhart \& Russell (1966).

Embora o teste $\mathrm{t}$ tenha confirmado as hipóteses $\left(H_{0}: \beta_{1 i}=1\right),\left(H_{0}: \beta_{2 i}=0\right)$ e $\left(H_{0}:\left(\beta_{1 i}+\beta_{2 i}\right)=1\right)$, a aplicação da regressão bissegmentada e sua interpretação se mostrou coerente com o desempenho das cultivares quando foram analisados os coeficientes em valores absolutos. As cultivares EMBRAPA 28, EMBRAPA 30, EMBRAPA 9, MT/BR-45, FT-Estrela, EMBRAPA 31, IAC-8, MT/BR-52, MT/BR-50 e MT/BR-51 apresentaram $\left(\beta_{1 \mathrm{i}}+\beta_{2 \mathrm{i}}\right)<1$ (Tabela 8 ), sugerindo, assim, uma baixa taxa de resposta à melhoria das condições ambientais (cultivares não responsíveis) e uma adaptação específica a ambientes de baixa produtividade. Destas cultivares, apenas as cultivares EMBRAPA 28, EMBRAPA 30, EMBRAPA 9 e MT/BR-51 apresentaram estabilidade acima da média nos ambientes desfavoráveis $\beta_{1}<1$ (Tabela 8), sendo que as cultivares EMBRAPA $28 \mathrm{e}$ EMBRAPA 9 apresentaram baixo rendimento, portanto pobremente adaptados, ao passo que a cultivar MT/BR-51 apresentou alta produtividade média $(3.035,17 \mathrm{~kg} / \mathrm{ha})$. As demais cultivares apresentaram estabilidade abaixo da média nos ambientes desfavoráveis $\left(\beta_{1}>1\right)$, e produtividade ao redor da média, portanto, pobremente adaptados às condições do experimento.

Os genótipos MT/BR-49, BR/EMGOPA-314, EMBRAPA 20, MGBR-46, MT/BR-47, EMGOPA-313 Rch, EMGOPA-308, MT/BR-55, Milionária, FT-Cristalina e MT/BR-53 apresentaram-se responsíveis às melhorias das condições ambientais e adaptados a ambientes de alta produtividade $\left(\beta_{1 \mathrm{i}}+\beta_{2 \mathrm{i}}\right)>1$, sendo que MT/BR-49, EMGOPA-308, FT-Cristalina, MT/BR-53, EMBRAPA 20 e EMGOPA-314 apresentaram estabilidade acima da média nos ambientes desfavoráveis $\beta_{1}<1$. Entre estas cultivares apenas a Milionária apresentou pequeno potencial produtivo $(1.977,17 \mathrm{~kg} / \mathrm{ha})$. 
Tabela 8. Estimativa dos coeficientes $\beta_{0}, \beta_{1 \mathrm{i}}$ e $\beta_{2 \mathrm{i}}$ de 21 cultivares de soja, utilizando o método descrito por Cruz et al. (1989) para rendimento de grãos (kg/ha) em 5 épocas de semeadura $(5 / 11 / 96,15 / 11 / 96,30 / 11 / 96,15 / 12 / 96$ e 1/1/97). Vilhena, RO.

\begin{tabular}{|c|c|c|c|c|c|c|c|c|}
\hline Cultivar & $\beta_{0}$ & $\beta_{1 \mathrm{i}}$ & $\mathrm{t}\left(\beta_{1 \mathrm{i}}=1\right)$ & $\beta_{2 \mathrm{i}}$ & $\mathrm{t}\left(\beta_{2}=0\right)$ & $\beta_{1 i}+\beta_{2 i}$ & $\mathrm{t}\left(\beta_{1 \mathrm{i}}+\beta_{2 \mathrm{i}}\right)=1$ & $\mathrm{R}^{2}(\%)$ \\
\hline MT/BR-55 & $3.250,89$ & 1,15 & $0,57^{\mathrm{ns}}$ & 0,70 & $0,34^{\mathrm{ns}}$ & 1,85 & $0,43^{\mathrm{ns}}$ & 98,33 \\
\hline MT/BR-53 & $3.050,17$ & 0,75 & $-0,98^{\mathrm{ns}}$ & 0,33 & $0,16^{\mathrm{ns}}$ & 1,07 & $0,04^{\mathrm{ns}}$ & 83,96 \\
\hline MT/BR-50 & $3.038,92$ & 1,14 & $0,54^{\mathrm{ns}}$ & $-1,14$ & $-0,55^{\mathrm{ns}}$ & 0,00 & $-0,51^{\mathrm{ns}}$ & 59,84 \\
\hline MT/BR-51 & $3.035,17$ & 0,88 & $-0,47^{\mathrm{ns}}$ & 0,06 & $0,03^{\mathrm{ns}}$ & 0,94 & $-0,03^{\text {ns }}$ & 98,55 \\
\hline BR/EMGOPA-314 & $2.936,68$ & 0,92 & $-0,32^{\mathrm{ns}}$ & 3,11 & $1,50^{\mathrm{ns}}$ & 4,03 & $1,54^{\mathrm{ns}}$ & 96,85 \\
\hline FT-Cristalina & $2.902,92$ & 0,87 & $-0,50^{\mathrm{ns}}$ & 0,30 & $0,14^{\mathrm{ns}}$ & 1,17 & $0,09^{\mathrm{ns}}$ & 67,51 \\
\hline EMGOPA-308 & $2.901,98$ & 0,70 & $-1,18^{\mathrm{ns}}$ & 1,51 & $0,73^{\mathrm{ns}}$ & 2,21 & $0,61^{\mathrm{ns}}$ & 97,12 \\
\hline MT/BR-52 & $2.830,69$ & 1,11 & $0,44^{\mathrm{ns}}$ & $-4,10$ & $-1,98 *$ & $-2,99$ & $-2,03 *$ & 97,37 \\
\hline EMGOPA-313 & $2.793,18$ & 1,35 & $1,35^{\mathrm{ns}}$ & 1,08 & $0,52^{\mathrm{ns}}$ & 2,43 & $0,73^{\mathrm{ns}}$ & 97,25 \\
\hline MT/BR-49 & $2.750,97$ & 0,57 & $-1,65^{\mathrm{ns}}$ & 3,45 & $1,67^{\mathrm{ns}}$ & 4,03 & $1,54^{\mathrm{ns}}$ & 93,13 \\
\hline MT/BR-47 & $2.750,97$ & 1,32 & $1,26^{\mathrm{ns}}$ & 1,11 & $0,54^{\mathrm{ns}}$ & 2,43 & $0,73^{\mathrm{ns}}$ & 95,69 \\
\hline IAC-8 & $2.707,82$ & 1,33 & $1,27^{\mathrm{ns}}$ & $-3,40$ & $-1,64^{\mathrm{ns}}$ & $-2,08$ & $-1,56^{\mathrm{ns}}$ & 90,12 \\
\hline EMBRAPA 30 & $2.696,57$ & 0,60 & $-1,54^{\mathrm{ns}}$ & $-0,25$ & $-0,12^{\mathrm{ns}}$ & 0,36 & $-0,33^{\text {ns }}$ & 56,60 \\
\hline MT/BR-45 & $2.667,49$ & 1,08 & $0,30^{\mathrm{ns}}$ & $-1,53$ & $-0,74^{\mathrm{ns}}$ & $-0,45$ & $-0,74^{\mathrm{ns}}$ & 94,76 \\
\hline FT-Estrela & $2.651,55$ & 1,14 & $0,55^{\mathrm{ns}}$ & $-1,66$ & $-0,80^{\mathrm{ns}}$ & $-0,52$ & $-0,77^{\mathrm{ns}}$ & 97,39 \\
\hline EMBRAPA 31 & $2.648,74$ & 1,48 & $1,88^{\mathrm{ns}}$ & $-3,40$ & $-1,64^{\mathrm{ns}}$ & $-1,92$ & $-1,48^{\mathrm{ns}}$ & 83,43 \\
\hline MGBR-46 & $2.640,29$ & 1,08 & $0,31^{\mathrm{ns}}$ & 1,81 & $0,87^{\mathrm{ns}}$ & 2,89 & $0,96^{\mathrm{ns}}$ & 88,71 \\
\hline EMBRAPA 20 & $2.519,30$ & 0,93 & $-0,27^{\mathrm{ns}}$ & 2,83 & $1,37^{\mathrm{ns}}$ & 3,77 & $1,41^{\mathrm{ns}}$ & 79,68 \\
\hline EMBRAPA 28 & $2.493,97$ & 0,76 & $-0,92^{\mathrm{ns}}$ & $-0,28$ & $-0,14^{\mathrm{ns}}$ & 0,49 & $-0,26^{\mathrm{ns}}$ & 67,59 \\
\hline EMBRAPA 9 & $2.358,91$ & 0,74 & $-1,03^{\mathrm{ns}}$ & $-0,93$ & $-0,45^{\mathrm{ns}}$ & $-0,20$ & $-0,61^{\mathrm{ns}}$ & 92,26 \\
\hline Milionária & $1.977,17$ & 1,10 & $0,39^{\mathrm{ns}}$ & 0,39 & $0,19^{\mathrm{ns}}$ & 1,49 & $0,25^{\mathrm{ns}}$ & 80,13 \\
\hline
\end{tabular}

nsNão-significativo. *Significativo a $5 \%$ de probabilidade.

As estimativas de $\left(\beta_{1 \mathrm{i}}+\beta_{2 \mathrm{i}}\right)$ referentes às cultivares MT/BR-53 e MT/BR-51 apresentaram valores próximos a 1 , indicando apresentarem estabilidade média nos ambientes favoráveis e adaptabilidade acima da média nos ambientes desfavoráveis $\left(\beta_{1 \mathrm{i}}\right)<1$; em ambos os casos, estes coeficientes estão associados à alta produtividade. Entre todas as cultivares testadas, apenas MT/BR-50 apresentou estabilidade absoluta $\left(\beta_{1 i}+\beta_{2 i}\right)=0$ nos ambientes de alta produtividade e estabilidade abaixo da média nos ambientes desfavoráveis $\left(\beta_{1 \mathrm{i}}\right)>1$, sempre associados à alta produtividade.

Na Tabela 9, são apresentadas as estimativas de $\beta_{0}, \beta_{1}$ e $\sigma^{2}$ (di). Segundo o modelo de Eberhart \& Russell (1966), a cultivar ideal é a que apresenta produção média alta, coeficiente de regressão igual à unidade e com pequeno desvio de regressão, ou seja, a cultivar com resposta positiva à melhoria das condições ambientais $\left(\beta_{1}=1\right)$ e com comportamento previsível $\left(\sigma^{2} d_{i}=0\right)$. Todas as estimativas de $\left(\beta_{1 i}\right)$ não apresentaram diferenças significativas da unidade
Tabela 9. Estimativa das variâncias dos coeficientes $\beta_{0}$, $\beta_{1 \mathrm{i}}$ e $\beta_{2 \mathrm{i}}$ e $\beta_{1}+\beta_{2}$, suas covariâncias e correlações utilizando o método descrito por Cruz et al. (1989).

\begin{tabular}{lrccc}
\hline Coeficiente & \multicolumn{1}{c}{$\sigma^{2} \mathrm{x}$} & $\sigma^{2} \mathrm{y}$ & $\mathrm{COV}(\mathrm{X}, \mathrm{Y})$ & Correlação \\
\hline$\beta_{0} \mathrm{e} \beta_{1}$ & $75.343,170$ & 0,07 & $-1,14$ & $-0,02$ \\
$\beta_{0}$ e $\beta_{2}$ & $75.343,170$ & 4,29 & 39,21 & 0,07 \\
$\beta_{1}$ e $\beta_{2}$ & 0,067 & 4,29 & $-0,24$ & $-0,45$ \\
$\beta_{1}$ e $\beta_{1}+\beta_{2}$ & 0,067 & 3,87 & $-0,17$ & $-0,34$ \\
$\beta_{0}$ e $\beta_{1}+\beta_{2}$ & $75.343,170$ & 3,87 & 38,07 & 0,07 \\
\hline
\end{tabular}

$\beta_{1}=1(\mathrm{P}<0,05)$, o que indica boa adaptabilidade geral em todas as cultivares; entretanto, quando foi analisada a significância dos desvios de regressão, as cultivares MT/BR-50 e EMBRAPA 31 apresentaram desvios de regressão significativos $(\mathrm{P}<0,05)$, indicando, desta maneira, que as variações de produção observadas nestas cultivares, nos diferentes ambientes, não foram apropriadamente representadas pelo modelo linear de regressão. 
Tabela 10. Análise de variância para rendimento de grãos em $\mathrm{kg} / \mathrm{ha}$, utilizando o método de Eberhart \& Russell (1966), com soma de quadrado do desvio para cada genótipo. Vilhena, RO.

\begin{tabular}{|c|c|c|c|c|}
\hline Fonte de variação & GL & Soma de quadrados & Quadrados médios & $\mathrm{R}^{2}$ \\
\hline Ambiente (A) & 4 & $30.854 .254,000$ & $7.713 .563,500^{* *}$ & \\
\hline Genótipo (G) & 20 & $22.603 .008,000$ & $1.130 .150,375^{* *}$ & \\
\hline Int GxA & 80 & $9.850 .322,000$ & $123.129,023^{\text {ns }}$ & \\
\hline $\mathrm{Amb} / \mathrm{Gen}$ & 84 & $40.704 .576,000$ & $484.578,281 * *$ & \\
\hline Amb.linear & 1 & $30.854 .204,00$ & $30.854 .204,00$ & \\
\hline G x amb li & 20 & $1.712 .276,00$ & $85.613,80$ & \\
\hline Dev.comb & 63 & $8.138 .161,00$ & $129.177,16$ & \\
\hline Desv g-1 & 3 & $49.241,75$ & $16.413,92^{\mathrm{ns}}$ & 97,58 \\
\hline Desv $\mathrm{g}-2$ & 3 & $162.982,50$ & $54.327,50^{\text {ns }}$ & 83,63 \\
\hline Desv g-3 & 3 & $1.291 .019,00$ & $430.339,66 *$ & 58,56 \\
\hline Desv g-4 & 3 & $16.968,38$ & $5.656,13^{\text {ns }}$ & 98,53 \\
\hline Desv g-5 & 3 & $351.974,38$ & $117.324,79^{\mathrm{ns}}$ & 80,12 \\
\hline Desv g-6 & 3 & $549.348,50$ & $183.116,17^{\text {ns }}$ & 67,35 \\
\hline Desv g-7 & 3 & $95.206,31$ & $31.735,44^{\mathrm{ns}}$ & 89,09 \\
\hline Desv g- 8 & 3 & $570.799,00$ & $190.266,33^{\mathrm{ns}}$ & 73,05 \\
\hline Desv g-9 & 3 & $115.515,50$ & $38.505,17^{\mathrm{ns}}$ & 96,00 \\
\hline Desv g-10 & 3 & $437.351,19$ & $145.783,73^{\text {ns }}$ & 58,45 \\
\hline Desv g-11 & 3 & $159.569,75$ & $53.189,92^{\mathrm{ns}}$ & 94,36 \\
\hline Desv g-12 & 3 & $647.801,50$ & $215.933,83^{\text {ns }}$ & 78,11 \\
\hline Desv g-13 & 3 & $405.805,00$ & $135.268,33^{\mathrm{ns}}$ & 56,40 \\
\hline Desv g-14 & 3 & $164.334,50$ & $54.778,17^{\mathrm{ns}}$ & 90,69 \\
\hline Desv g-15 & 3 & $135.154,25$ & $45.051,42^{\mathrm{ns}}$ & 93,01 \\
\hline Desv g-16 & 3 & $1.006 .349,50$ & $335.449,84 *$ & 74,43 \\
\hline Desv g-17 & 3 & $347.379,63$ & $115.793,21^{\mathrm{ns}}$ & 84,13 \\
\hline Desv g-18 & 3 & $677.263,13$ & $225.754,38^{\text {ns }}$ & 68,07 \\
\hline Desv g-19 & 3 & $408.037,38$ & $136.012,45^{\text {ns }}$ & 67,40 \\
\hline Desv g-20 & 3 & $91.890,38$ & $30.630,13^{\mathrm{ns}}$ & 89,11 \\
\hline Desv g-21 & 3 & $454.169,13$ & $151.389,70^{\text {ns }}$ & 79,92 \\
\hline Resíduo & 200 & $24.527 .410,00$ & $122.637,058$ & \\
\hline
\end{tabular}

nsNão-significativo. * e **Significativo a $5 \%$ e $1 \%$ de probabilidade, respectivamente.

Tabela 11. Estimativa dos coeficientes $\beta_{0}, \beta_{1 \mathrm{i}}$ e $\sigma^{2}\left(\mathrm{~d}_{\mathrm{i}}\right)$ utilizando o método de Eberhart \& Russell (1966), para rendimento de grãos $(\mathrm{kg} / \mathrm{ha})$, na semeadura realizada em 5/11/96, 15/11/96, 30/11/96, 15/12/96 e 1/1/97. Vilhena, RO.

\begin{tabular}{|c|c|c|c|c|}
\hline \multirow[t]{2}{*}{ Cultivar } & \multicolumn{4}{|c|}{ Rendimento de grãos } \\
\hline & $\beta_{0}$ & $\beta_{1 \mathrm{ii}}$ & $\mathrm{T}\left(\beta_{1 \mathrm{i}}=1\right)$ & $\sigma^{2}\left(d_{i}\right)$ \\
\hline MT/BR-51 & $3.035,16$ & 0,88 & $-0,42^{\mathrm{ns}}$ & $-38.993,64$ \\
\hline MT/BR-55 & $3.250,89$ & 1,16 & $0,56^{\mathrm{ns}}$ & $-35.407,71$ \\
\hline EMBRAPA 9 & $2.358,91$ & 0,72 & $-0,98^{\mathrm{ns}}$ & $-30.668,98$ \\
\hline EMGOPA-308 & $2.901,98$ & 0,73 & $-0,94^{\mathrm{ns}}$ & $-30.300,54$ \\
\hline EMGOPA-313 Rch & $2.793,18$ & 1,37 & $1,29^{\text {ns }}$ & $-28.043,96$ \\
\hline FT-Estrela & $2.651,55$ & 1,11 & $0,37^{\mathrm{ns}}$ & $-25.861,88$ \\
\hline MT/BR-47 & $2.750,97$ & 1,35 & $1,20^{\mathrm{ns}}$ & $-23.149,05$ \\
\hline MT/BR-53 & $3.050,17$ & 0,75 & $-0,86^{\mathrm{ns}}$ & $-22.769,85$ \\
\hline MT/BR-45 & $2.667,49$ & 1,04 & $0,15^{\mathrm{ns}}$ & $-22.619,63$ \\
\hline MGBR-46 & $2.640,29$ & 1,12 & $0,41^{\mathrm{ns}}$ & $-2.281,28$ \\
\hline BR/EMGOPA-314 & $2.936,68$ & 0,98 & $-0,06^{\mathrm{ns}}$ & $-1.770,75$ \\
\hline EMBRAPA 30 & $2.696,57$ & 0,60 & $-1,39^{\mathrm{ns}}$ & $4.210,43$ \\
\hline EMBRAPA 28 & $2.493,97$ & 0,76 & $-0,84^{\mathrm{ns}}$ & $4.458,47$ \\
\hline MT/BR-49 & $2.750,97$ & 0,65 & $-1,22^{\mathrm{ns}}$ & $7.715,56$ \\
\hline Milionária & $1.977,17$ & 1,11 & $0,38^{\mathrm{ns}}$ & $9.584,22$ \\
\hline FT-Cristalina & $2.902,91$ & 0,88 & $-0,42^{\mathrm{ns}}$ & $20.159,70$ \\
\hline MT/BR-52 & $2.830,69$ & 1,03 & $0,09^{\mathrm{ns}}$ & $22.543,09$ \\
\hline IAC-8 & $2.707,82$ & 1,25 & $0,88^{\mathrm{ns}}$ & $31.098,93$ \\
\hline EMBRAPA 20 & $2.519,30$ & 0,99 & $-0,03^{\text {ns }}$ & $34.372,44$ \\
\hline EMBRAPA 31 & $2.648,73$ & 1,41 & $1,43^{\mathrm{ns}}$ & $70.937,59$ \\
\hline MT/BR-50 & $3.038,91$ & 1,11 & $0,40^{\mathrm{ns}}$ & $102.567,54$ \\
\hline$\overline{\sigma^{2}\left(\beta_{0}\right)}$ & $8.175,804$ & & & \\
\hline$\sigma^{2}\left(\beta_{1}\right)$ & $8,346713 \mathrm{E}-02$ & & & \\
\hline Correlação $\left(\beta_{0}\right.$ e $\left.\beta_{\text {li }}\right)$ & $-4,631216 \mathrm{E}-03$ & & & \\
\hline
\end{tabular}

\section{Conclusões}

1. Os métodos utilizados são coerentes entre si e permitem identificar, entre as cultivares avaliadas, as de maior estabilidade e adaptabilidade.

2. A não-significância das hipóteses $\left(\mathrm{H}_{0}: \beta_{1 \mathrm{i}}=1\right)$, $\left(\mathrm{H}_{0}: \beta_{2 \mathrm{i}}=0\right)$ e $\left(\mathrm{H}_{0}:\left(\beta_{1 \mathrm{i}}+\beta_{2 \mathrm{i}}\right)=1\right)$, no modelo de Cruz et al., indica que o comportamento produtivo das cultivares de soja ao longo das épocas de plantio é melhor representado pelo modelo linear proposto por Eberhart \& Russell.

3. Os coeficientes obtidos pelo método da regressão linear de Eberhart \& Russell apresentam uma gradação contínua de valores, mas nenhum destes coeficientes apresentam significância da hipótese $\left(\mathrm{H}_{0}: \beta_{1 \mathrm{i}}=1\right)$ pelo teste $\mathrm{t}_{5 \%, 200}$; as cultivares que apresentam a maior estimativa referente à variância dos desvios são MT/BR-50 e EMBRAPA 31.

4. As épocas de plantio realizadas entre 5/11/96 e $15 / 11 / 96$ se mostram as mais favoráveis para obtenção de produtividades de soja acima de $2.700 \mathrm{~kg} / \mathrm{ha}$.

\section{Agradecimentos}

Ao Sr. Ildeu Alves Ribeiro, assistente de operações, pela colaboração durante a condução da pesquisa.

\section{Referências}

ALLIPRANDINI, L. F.; TOLEDO, J. F. F.; FONSECA JÚNIOR, N.; ALMEIDA, L.; KIIHL, S. Efeitos da interação genótipo $\mathrm{x}$ ambiente sobre a produtividade da soja no Estado do Paraná. Pesquisa Agropecuária Brasileira, Brasília, v. 29, n. 9, p. 1433-1444, set. 1994.

CRUZ, C. D. Programa GENES: aplicativo computacional em genética e estatística. UFV : Viçosa, 1997. disquete.

CRUZ, C. D.; TORRES, R. A.; VENCOVSKY, R. An alternative approach to the stability analysis proposed by Silva and Barreto. Revista Brasileira de Genética, Ribeirão Preto, v. 12, p. 567-580, 1989.

EASTON, H. S.; CLEMENTS, R. J. The interaction of wheat genotypes with a specific factor of the environment. Journal of Agricultural Science, Cambridge, GrãBretanha, v. 80, p. 43-52, 1973.

EBERHART, S. A.; RUSSELL, W. A. Stability parameters for comparing varieties. Crop Science, Madison, v. 6, p. 3640, 1966. 
FINLAY, K. W.; WILKINSON, G. N. The analysis of adaptation in plant-breeding programme. Australian Journal of Agricultural Research, Collingwood, v. 14, p. 742-754, 1963.

FREEMAN, G. H.; PERKINS, J. M. Environmental and genotype-environmental components of variability. VIII. Relations between genotypes grown in different environments and measures of these environments. Heredity, v. 27, p. 15-23, 1971

LIN, C. S.; BINNS, M. R.; LEFKOVITCH, L. P. Stability analysis: Where do we stand? Crop Science, Madison, v. 26 , p. 894-900, 1986.

PERKINS, J. M.; JINKS, J. L. Environmental and genotype-environmental components of variability. III. Multiple lines and crosses. Heredity, Oxford, v. 23, p. $339-356,1968$.

PLAISTED, R. L.; PETERSON, L. C. A technique for evaluating the ability of selections to yield consistently in different locations or seasons. American Potato Journal, Orono, v. 36, p. 381-385, 1959.

RAMALHO, M. P.; SANTOS, J. B.; ZIMMERMANN, M. J. Interação dos genótipos por ambientes. In:

Genética quantitativa em plantas autógamas: aplicações ao melhoramento do feijoeiro. Goiânia : Ed. da UFG 1993. p. $137-170$.
SAKIYAMA, N. S.; SEDIYAMA, T.; SEDIYAMA, C. S.; REIS, M. S. Interação genótipo $x$ ambiente e efeitos na escolha de localidades para avaliação de linhagens de soja. (Glycine max (L.) Merrill). Revista Ceres, Viçosa, v. 35, n. 201, p. 486-493, 1988.

SILVA, J. G. C. Análise da adaptabilidade através de regressão linear segmentada. I. Fundamentos. Pesquisa Agropecuária Brasileira, Brasília, v. 30, n. 4, p. 435-448, abr. 1995.

SILVA, J. G. C.; BARRETO, J. N. An application of segmented linear regression to the study of genotype $\mathrm{x}$ environment interaction. Biometrics, Washington, v. 41, n. 4, p. 1093, 1986.

TAI, G. C. C. Genotype stability analysis and its a application to potato regional trials. Crop Science, Madison, v. 11, p. 184-190, 1971.

VERMA, M. M.; CHAHAL, G. S.; MURTY, B. R. Limitations of conventional regression analysis: a proposed modification. Theoretical and Applied Genetics, Berlin, v. 53, p. 89-91, 1978.

WRICKE, G. Zur Berechnung der Ökovalenz bei Sommerweizen und Hafer. Zeitschrift für Pflanzenzüchtung, Berlin, v. 52, p. 127-138, 1965. 